\title{
NORMAL SUBGROUPS OF MONOMIAL GROUPS
}

R. B. CROUCH AND W. R. SCOTT

1. Introduction. Let $U$ be a set, $o(X)=B=\boldsymbol{\aleph}_{u}, u \geqq 0$, where $o(U)$ means the number of elements of $U$. Let $H$ be a fixed group. A monomial substitution $y$ is a transformation that maps every $x$ of $U$ in a one-to-one fashion into an $x$ of $U$ multiplied by an element $h_{x}$ of $H$. Multiplication of substitutions means successive applications. The set of all monomial substitutions forms a group $\Sigma$. Ore [1] has studied this group for finite $U$, and some of his results have been generalized to general $U$ in [3].

The normal subgroups for one subgroup of $\Sigma$ have been determined [3]. This paper extends those results to the extent of determining the structure of all normal subgroups of various subgroups of $\Sigma$ in a rather general case. These results are stated in Theorems 1, 2, 3, $4,5,6$.

2. Definitions. Let $d$ be the cardinal of the integers. Let $B$ be an infinite cardinal; $B^{+}$, the successor of $B ; U$, a set such that $o(U)=B$; and $C$ such that $d<C \leqq B^{+}$. Let $H$ be a fixed group and $e$ the identity of $H$. Denote by $\Sigma=\Sigma(H, B, d, C)$ the monomial group of $U$ over $H$ whose elements are of the form

$$
y=\left(\begin{array}{cc}
\cdots, & x_{\epsilon}, \cdots \\
\cdots, & h_{\epsilon} x_{i_{\epsilon}}, \cdots
\end{array}\right)
$$

where only a finite number of the $h_{\epsilon}$ are not $e$ and the number of $x$ not mapped into themselves is less than $C$. Any element of $\Sigma$ may be written in the form

$$
y=\left(\begin{array}{cc}
\cdots, & x_{\epsilon}, \cdots \\
\cdots, & h_{\epsilon} x_{\epsilon}, \cdots
\end{array}\right)\left(\begin{array}{cc}
\cdots, & x_{\epsilon}, \cdots \\
\cdots, e x_{i_{\epsilon}}, \cdots
\end{array}\right)
$$

or $y=v s$ where $v$ sends every $x$ into itself, every $h$ of $s$ is $e$. Elements of the form of

$$
v=\left(\begin{array}{cc}
\cdots, & x_{\epsilon}, \cdots \\
\cdots, & h_{\epsilon} x_{\epsilon}, \cdots
\end{array}\right)=\left\{\cdots, h_{\epsilon}, \cdots\right\}
$$

are multiplications and all such elements form a normal subgroup,

Presented to the Society, December 28, 1956; received by the editors March 18, 1956 and, in revised form, December 22, 1956. 
the basis group $V(B, d)=V$ of $\Sigma$. The $h_{\epsilon}$ of $y$ are called the factors of $y$. Elements of the form of $s$ are permutations and all such elements form a subgroup, the permutation group, $S(B, C)=S$ of $\Sigma(H ; B, d, C)$. Cycles of $s$ will also be written as $\left(x_{1}, \cdots, x_{n}\right)$ and $\left(\cdots, x_{-1}, x_{0}\right.$, $\left.x_{1}, \cdots\right)$. Baer [2] has shown that the normal subgroups of $S(B, C)$ are the alternating group, $A=A(B, d)$, and $S(B, D)$ where $d \leqq D \leqq C$. Let $E$ be the identity of $\Sigma, I$ the identity of $S$.

\section{Normal subgroups of $\Sigma$ contained in the basis group.}

Theorem 1. Let $G_{1} \subset G$ be normal subgroups of $H$ such that $G / G_{1}$ belongs to the center of $H / G_{1}$. Let $N$ be the set of multiplications with a finite number of different from e factors subject to the conditions that the factors are in $G$ and their product, in any order, belongs to $G_{1} . N$ is a normal subgroup of $\Sigma$ contained in the basis group. Conversely, let $N$ be a normal subgroup of $\Sigma$ contained in the basis group. For every $v$ of $N$ form all possible products of its nonidentity factors. This set $G_{1}$ is a normal subgroup of $H$. The set $G$ consisting of every element of $h$ that occurs as a factor in any $v$ of $N$ is also a normal subgroup of $H$. Further, $G / G_{1}$ belongs to the center of $H / G_{1}$.

Proof. It is clear that $N$ is a subgroup. Let $y_{1}=v_{1} s_{1}$ be any element of $\Sigma$. Then $y_{1} v y_{1}^{-1}=v_{1} s_{1} v s_{1}^{-1} v_{1}^{-1}=v_{1} v_{2} v_{1}^{-1}$ where $v_{2}$ belongs to $N$. Since $G / G_{1}$ is in the center of $H / G_{1}$ the product of the factors of $v_{1} v_{2} v_{1}^{-1}$ is in $G_{1}$ and $N$ is normal in $\Sigma$.

If $N$ is normal in $\Sigma$ then an argument similar to that in [3, pp. 204207] shows it has the structure described in the theorem.

4. Normal subgroups of $\Sigma$ not contained in the basis group. The problem of finding all normal subgroups of $\Sigma$ will be concluded by finding those normal subgroups not in $V$.

Lemma 1. Let $M$ be normal in $\Sigma, V D M$. Then $N=M \cap V$ is normal in $\Sigma$ and $G=H$, i.e., the factors in any fixed position run through $H$ and $H / G_{1}$ is Abelian.

Proof. Choose $y=v s \epsilon M$ such that $s \neq I$. Let $v=\left\{k_{1}, k_{2}, \cdots\right\}$ be arbitrary in $V$. Then the multiplication $y^{-1} v^{-1} y v$ is in $M$. Let $s$ send $x_{\epsilon}$ into $x_{i_{\epsilon}}$ with $\epsilon \neq i_{\epsilon}$. The factor occurring in the position occupied by $x_{i_{\epsilon}}$ is $h_{\epsilon}^{-1} k_{\epsilon}^{-1} h_{\epsilon} k_{i_{\epsilon}}$. Since $v$ is arbitrary and $i_{\epsilon} \neq \epsilon$ we may choose the factors $k_{\epsilon}^{-1}, k_{i_{\epsilon}}$ in such a way that the factor above is arbitrary in $H$.

Lemma 2. Let $M$ be normal in $\Sigma, V D M$. Then $P=M \cap S$ is normal in $S$ and $P \neq E$.

Proof. Let $y$ be an element of $M$ and $y=v s$ where $s \neq 1$. Since $y$ 
has only a finite number of different from $e$ factors, $M$ must contain a $y^{\prime}$ conjugate to $y$ where the finite cycles of $y$ have been written in normal form, $\left[1\right.$, p. 20]. If $y$ contains an infinite cycle then $y^{\prime}$ contains an infinite cycle in the form,

$$
\left(\begin{array}{l}
\cdots, x_{-1}, x_{0}, x_{1}, \cdots \\
\cdots, x_{0}, g x_{1}, x_{2}, \cdots
\end{array}\right) .
$$

If $y^{\prime}$ contains a finite cycle then [1, pp. 35-36] $M$ contains a permutation. If $y^{\prime}$ contains only infinite cycles let $s_{1}=(2,3)$. Then the commutator

$$
\begin{aligned}
& \left(y^{\prime}\right)^{-1} s_{1} y^{\prime} s_{1}^{-1} \\
& \quad=\left(\begin{array}{lr}
\cdots, x_{-1}, x_{0}, & x_{1}, x_{2}, x_{3}, x_{4}, x_{5}, \cdots \\
\cdots, & x_{-1}, x_{0}, g^{-1} g x_{1}, x_{3}, x_{4}, x_{2}, x_{5}, \cdots
\end{array}\right)=(2,3,4)
\end{aligned}
$$

belongs to $M$.

THEOREM 2. If $M=N \cup P$, where $N$ is as in Lemma 1 and $P$ is a normal subgroup of $S$, then $M$ is normal in $\Sigma$.

PROof. Let $y=v s$ be any element of $M$. Let $y_{1}=v_{1} s_{1}$ be any element of $\Sigma$. Then $y_{1} y y_{1}^{-1}=y_{1} v y_{1}^{-1} v_{1} s_{1} s s_{1}^{-1} v_{1}^{-1}=v_{2} v_{1} s_{2} v_{1}^{-1}$ where $v_{2} \in N, s_{2} \in P$ since $N$ is normal in $\Sigma$ and $P$ is normal in $S$. It is sufficient to show $v_{1} s_{2} v_{1}^{-1}$ is in $M$. A computation shows

$$
\begin{aligned}
v_{1} s_{2} v_{1}^{-1} & =\left\{h_{1}, \cdots, h_{\epsilon}, \cdots\right\}\left(\begin{array}{c}
x_{1}, \cdots, x_{\epsilon}, \cdots \\
x_{i_{1}}, \cdots, x_{i_{\epsilon}}, \ldots
\end{array}\right)\left\{h_{1}^{-1}, \cdots, h_{\epsilon}^{-1}, \cdots\right\} \\
& =\left\{h_{1} h_{i_{1}}^{-1}, \cdots, h_{\epsilon} h_{i_{\epsilon}}^{-1}, \cdots\right\}=s_{2}=v_{3} s_{2}
\end{aligned}
$$

where all but a finite number of the factors of $v_{3}$ are $e$. Since $H / G_{1}$ is Abelian the product of the factors of $v_{3}$ is in $G_{1}$. Therefore, $v_{3} \in N$, $s_{2} \in P$ and $M$ is normal in $\Sigma$.

Theorem 3. If $V D M, M$ normal in $\Sigma, M \cap S=P=S(B, D)$, $d \leqq D \leqq C, M \cap V=N$ then $M=N \cup P$.

Proof. Assume there exists $y \in M, y=v s$, and $s \notin P$. This means $s$ moves $D$ or more of the elements of $U$. Then $y$ is conjugate to $y^{\prime}$ of $M$ where $y^{\prime}$ in its cyclic normal form has $d$-cycles written in the form

$$
\left(\begin{array}{l}
\cdots, x_{-1}, x_{0}, x_{1}, \cdots \\
\cdots, x_{0}, g x_{1}, x_{2}, \cdots
\end{array}\right) .
$$

Construct $s_{1}$ as follows. For each $n$-cycle, $n \geqq 3$, of $y^{\prime}$ of the form 


$$
c=\left(\begin{array}{cc}
x_{1}, \cdots, & x_{n} \\
x_{2}, \cdots, a x_{1}
\end{array}\right)
$$

let $s_{1}$ have the cycle $\left(x_{1}, x_{2}\right)$. For each pair of 2-cycles

$$
\left(\begin{array}{cc}
x_{1}, & x_{2} \\
x_{2}, b x_{1}
\end{array}\right), \quad\left(\begin{array}{cc}
x_{3}, & x_{4} \\
x_{4}, c x_{3}
\end{array}\right)
$$

of $y^{\prime}$ let $s_{1}$ have the cycle $\left(x_{1}, x_{3}\right)\left(x_{2}\right)\left(x_{4}\right)$. If there is a 2-cycle

$$
\left(\begin{array}{cc}
x_{\alpha}, & x_{\beta} \\
x_{\beta}, & d x_{\alpha}
\end{array}\right)
$$

of $y^{\prime}$ left over let $s_{1}$ send $s_{\alpha}, s_{\beta}$ into themselves. For each $d$-cycle of $y^{\prime}$

$$
\left(\begin{array}{l}
\cdots, x_{-1}, x_{0}, x_{1}, \cdots \\
\cdots, x_{0}, g x_{1}, x_{2}, \cdots
\end{array}\right)
$$

let $s_{1}$ have the cycles

$$
\left(\begin{array}{l}
x_{0} \\
x_{0}
\end{array}\right)\left(\begin{array}{l}
x_{n}, x_{-n} \\
x_{-n}, x_{n}
\end{array}\right) \quad \text { for } n=1,2, \cdots
$$

Form the commutator $\left(y^{\prime}\right)^{-1} s_{1} y^{\prime}\left(s_{1}\right)^{-1}=y_{1}$ which is in $M$. For each $n$-cycle, $n \geqq 3$, of $y^{\prime}, y_{1}$ contains $\left(x_{1}, x_{2}, x_{3}\right)$. For each pair of 2-cycles $y_{1}$ contains $\left(x_{1}, x_{3}\right)\left(x_{2}, x_{4}\right)$. For each $d$-cycle of $y^{\prime}, y_{1}$ will contain

$$
\begin{aligned}
& \left(\begin{array}{lr}
\cdots, x_{-3}, x_{-2}, x_{-1}, & x_{0}, \quad x_{1}, x_{2}, x_{3}, \cdots \\
\cdots, x_{-5}, x_{-4}, x_{-3}, x_{-2}, g^{-1} g x_{-1}, x_{0}, x_{1}, \cdots
\end{array}\right) \\
& =\left(\begin{array}{c}
\cdots, x_{2}, x_{0}, x_{-2}, \cdots \\
\cdots, x_{0}, x_{-2}, x_{-4}, \cdots
\end{array}\right)\left(\begin{array}{l}
\cdots, x_{3}, x_{1}, x_{-1}, \cdots \\
\cdots, x_{1}, x_{-1}, x_{-3}, \cdots
\end{array}\right) .
\end{aligned}
$$

This shows that $M$ contains a permutation which moves the same number of $x$ 's as the $s$ of $y=v s$, contradicting $P=S(B, D)$.

If $y=v s \in M$ then since $s \in M, s^{-1}$ also belongs to $M$, and $y s^{-1}$ $=v \in M$.

THEOREM 4. Let $M \subset \Sigma, M$ normal in $\Sigma, M \cap S=A(B, d), M \cap V=N$ and $M / N \cong A(B, d)$. Then $M=N \cup A(B, d)$.

Proof. Since $M \supset(N \cup A)$ and $N \cup A$ is normal in $\Sigma$ it follows that $N \cup A$ is normal in $M$. Thus $N \cup A / N$ is normal in $M / N$ which is simple since $M / N \cong A(B, d)$. Therefore, $N \cup A=M$.

TheOREM 5. Let $M \subset \Sigma, M$ normal in $\Sigma, V D M, M \cap S=A, M \cap V$ $=N$, and $M / N$ not $\cong A$. Then $M=N \cup A \cup L$, where $L$ is the cyclic subgroup generated by 


$$
y=\left(\begin{array}{cc}
x_{1}, & x_{2} \\
x_{2}, & a x_{1}
\end{array}\right)
$$

with $a^{2}$ in $G_{1}$. If $L_{1}$ is the cyclic subgroup generated by

$$
y_{1}=\left(\begin{array}{ll}
x_{1}, & x_{2} \\
x_{2}, & b x_{1}
\end{array}\right),
$$

where $b^{2} \in G_{1}, M_{1}=N \cup A \cup L_{1}$ is $M$ if, and only if, $a$ and $b$ are in the same coset of $G_{1}$.

Proof. $M$ contains an element $y=v s$ with $s$ in $S(B, C), C \geqq d$, and $s \notin A$. Otherwise, for every $y=v s$ of $M, s \in A$. Then every element of $V \cup M$ would be of the form $y=v_{1} v s=v_{2} s$ and $(V \cup M) / V \cong A$. But $(V \cup M) / V \cong M / N$ not $\cong A$. Now if $y=v s$ with $s \in S(B, C)$ with $C>d$ the method used to prove Theorem 3 will lead to $M \cap S \neq A$, a contradiction. So assume $s \in S(B, d)$. The product of any 2 elements of $M$ outside $N \cup A$ is in $N \cup A$ since the permutation component is finite and even. Let $x_{1}, x_{2}$ be two elements $s$ leaves fixed. The permutations $s^{-1}\left(x_{1}, x_{2}\right)$ belong to $A=P \subset M$. Therefore, $y s^{-1}\left(x_{1}, x_{2}\right)=v s s^{-1}\left(x_{1}, x_{2}\right)$ belongs to $M$. There is an element $v_{1}$ in $N$ such that $v_{1} v\left(x_{1}, x_{2}\right)$ can be reduced to the form

$$
y=\left(\begin{array}{cc}
x_{1}, & x_{2} \\
x_{2}, & a x_{1}
\end{array}\right)
$$

because the factors of elements of $N$ are unrestricted except that the product is in $G_{1}$. This element squared is in $N \subset M$ so $a^{2} \in G_{1}$.

Theorem 6. Let $M=N \cup A \cup L$, where $N$ is as in Lemma 1 and $L$ is the cyclic group generated by

$$
y=\left(\begin{array}{cc}
x_{1}, & x_{2} \\
x_{2}, & a x_{1}
\end{array}\right)
$$

with $a^{2} \in G_{1}$. Then $M$ is normal in $\Sigma$.

Proof. It is sufficient to show $v s y(v s)^{-1}$ belongs to $M$ for all $v s$ of $\Sigma$ because $y^{2}$ belongs to $N$. This may be reduced to showing $s y s^{-1}$ and $v y v^{-1}$ belong to $M$. Let $s$ be arbitrary in $S$ and

Then

$$
s=\left(\begin{array}{l}
x_{i}, \cdots, x_{j}, \cdots \\
x_{1}, \cdots, x_{2}, \cdots
\end{array}\right) .
$$

$$
s y s^{-1}=\left(\begin{array}{cc}
x_{i}, & x_{j} \\
x_{j}, & a x_{i}
\end{array}\right) .
$$


But there exists an $s_{1}$ of $A$ such that

$$
s_{1}=\left(\begin{array}{c}
x_{i}, \cdots, x_{j}, \cdots \\
x_{1}, \cdots, x_{2}, \cdots
\end{array}\right) \text {. }
$$

But sys $s^{-1}=s_{1} y s_{1}^{-1}$ belongs to $(A \cup L \cup A) \subset M$. Let $v$ be arbitrary in $V$ and $v=\left\{h_{1}, \cdots, \quad h_{\epsilon}, \cdots\right\}$. The commutator $y^{-1} v y v^{-1}$ $=\left\{a^{-1} h_{2} a h_{1}^{-1}, h_{1} h_{2}^{-1}, e, \cdots\right\}$ belongs to $N \subset M$ if $a^{-1} h_{2} a h_{1}^{-1} h_{1} h_{2}^{-1}$, belongs to $G_{1}$. Since $H / G_{1}$ is Abelian the desired result follows.

Theorem 7. If $C=\boldsymbol{\aleph}_{n}$, where $n$ is finite, and if $H$ is finite then the number of normal subgroups of $\Sigma$ is finite.

Proof. This follows from the fact that there are only a finite number of choices of (1) normal subgroups of $S(B, C)$, (2) normal subgroups of $H,(3)$ cosets of $G_{1}$.

\section{BIBLIOGRAPHY}

1. O. Ore, Theory of monomial groups, Trans. Amer. Math. Soc. vol. 51 (1942) pp. 15-64.

2. R. Baer, Die Kompositionsreihe der Gruppe aller eineindeutigen Abbildungen einer unendlichen Reihe auf sich, Studia Math. vol. 5 (1934) pp. 15-17.

3. R. Crouch, Monomial groups, Trans. Amer. Math. Soc. vol. 80 (1955) pp. 187215 .

New Mexico College of Agriculture and Mechanical Arts, UNIVERSITY OF KANSAS AND

The Institute for Advanced Study 